\title{
Congenital adhesion band causing recurrent subacute intestinal obstruction in a virgin abdomen
}

\section{Dear Editor,}

Intestinal obstruction (IO) caused by malignancy and adhesion bands from previous surgery is common among adults. However, IO caused by congenital adhesion bands $(\mathrm{CAB})$ in the elderly is rare. We report a case of a 63-year-old man who presented with acute-on-chronic intestinal obstruction due to $\mathrm{CAB}$, which caused pseudointestinal malrotation.

Case presentation. A 63-year-old Chinese man with no past surgical history presented with severe abdominal pain, vomiting, abdominal distension and obstipation for the past 24 hours. He reported recurrent episodes of abdominal pain associated with nausea, vomiting and abdominal distension over the past 5 years that spontaneously resolved. Upon scrutiny of his medical records, it was discovered that he had presented on 3 previous occasions with similar symptoms and was conservatively treated for gastroenteritis with ileus.

He was haemodynamically stable and afebrile. Physical examination revealed a tense abdomen with mild central tenderness without signs of peritonism. There was no evidence of hernias and digital rectal examination revealed an empty rectum with no masses. Inflammatory markers were normal and abdominal radiograph showed dilated small bowel loops with multiple air-fluid levels, suggestive of intestinal obstruction. A computed tomography (CT) scan of the abdomen and pelvis was subsequently performed and revealed diffuse small bowel dilatation with fecalisation but no obvious transition point.

Trial of conservative management with nasogastric tube insertion, intravenous hydration and fasting over the next 48 hours showed no improvement and the patient was counselled for exploratory laparotomy.

On entry into the abdomen, clear serous fluid was seen with dilated but healthy small bowel. Interestingly, the caecum and appendix were seen in the left iliac fossa with an intraperitoneal ascending colon (Fig. 1). After extensive adhesiolysis, the duodenal-jejunal flexure was found in its correct anatomical location. Dense adhesions were found between the edge of the left lobe of the liver and small bowel, requiring wedge resection of the liver. Additionally, there was a $\mathrm{CAB}$

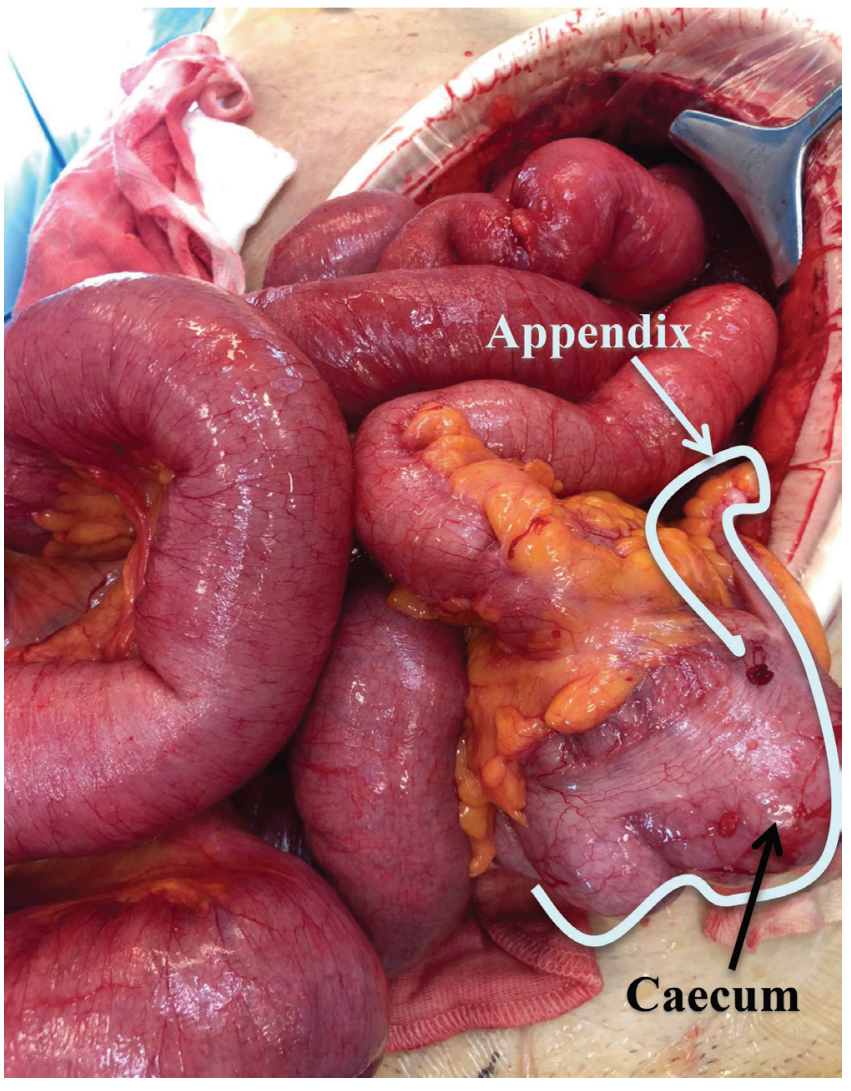

Fig. 1. Caecum and appendix were found in the left iliac fossa. There were no other signs of malrotation.

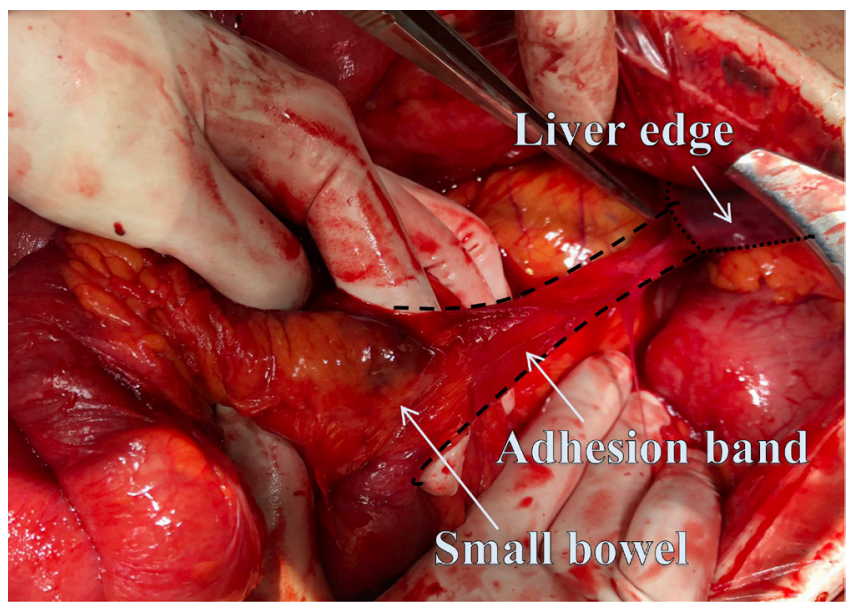

Fig. 2. Congenital adhesion band from small bowel to liver with resultant twist in the small bowel mesentery. 
from the root of the mesentery to the falciform ligament resulting in twisting of the small bowel mesentery (Fig. 2). The $\mathrm{CAB}$ and lack of fixation of the right colon to the posterior abdominal wall resulted in multiple sites of internal herniation of the small bowel, which were anchored at 3 points - right hypochondrium, anterior abdominal wall and edge of the left lobe of the liver. There were also multiple interloop adhesions (Fig. 3). All adhesion bands were ligated and the bowel returned to the abdomen in its normal configuration. Appendectomy was done to prevent future confusion in view of an intraperitoneal caecum, and the small bowel was subsequently decompressed via a cecostomy through the appendiceal stump. Postoperative recovery was uneventful.

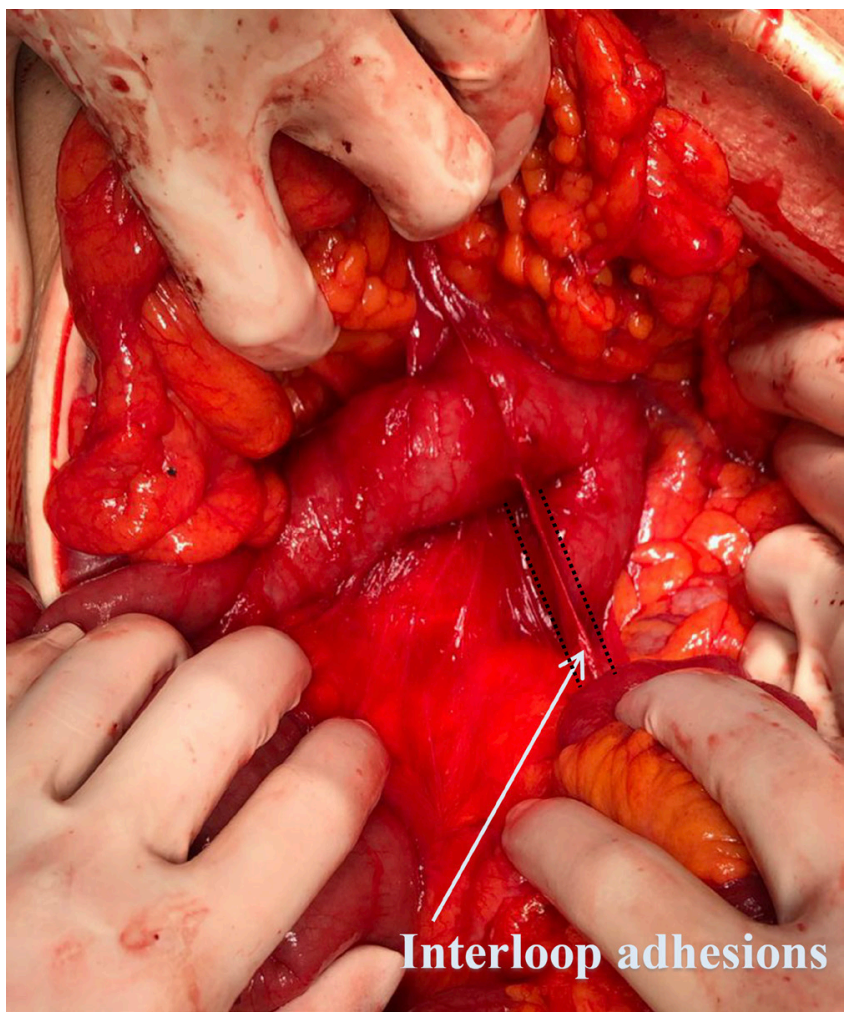

Fig. 3. Multiple interloop adhesions.

Patient and disease characteristics. $\mathrm{CAB}$ is a rare surgical entity with few case series and case reports in the literature. Its incidence remains unclear. It is an infrequent cause of $\mathrm{IO}$ in the paediatric population, and is extremely rare in adults..$^{1-5} \mathrm{CAB}$ can be found at various sites within the peritoneal cavity, such as those running between the terminal ileum and ascending colon. ${ }^{2}$ However, CABs are usually found at a single site, resulting in a single transition point. ${ }^{1-3}$ From our literature search, this is the first case of CAB with multiple sites of dense adhesions that resulted in the compression and entrapment of small bowel at multiple sites. There has also been no reported case of CAB that was so densely adherent to the liver edge that a small wedge resection of the liver was required to avoid bowel resection.

Diagnosis. Prior to this admission, the patient had been treated conservatively with success on multiple occasions without further evaluation. This is not uncommon in view of his non-specific clinical findings and spontaneous recovery. However, in patients with a virgin abdomen who present recurrently with intestinal obstruction, and with a waxing and waning clinical picture, we need to consider mechanical obstruction due to abnormal anatomy such as CAB. This is an important differential to consider especially when further imaging studies-such as water-soluble contrast fluoroscopic study or CT scans - do not reveal structural lesions that could account for the IO. CAB as a cause of IO can be difficult to diagnose because it is radiologically occult. CT scans will show diffusely dilated small bowel loops with occasionally identifiable transition points, and no further cause of intestinal obstruction will be found. ${ }^{6}$ As such, diagnosis of $\mathrm{CAB}$ as a cause of IO remains a diagnosis of exclusion that has to be considered when CT reveals an abrupt change in bowel calibre without evidence of another cause of obstruction. Therefore, despite $\mathrm{CAB}$ being a rare pathology, it is important to consider the differential of $\mathrm{CAB}$ in patients from all age groups who present with symptoms and signs of intestinal obstruction in the absence of previous abdominal surgeries and hernias. A high index of suspicion should be maintained even when patients appear clinically well with normal haematological results, as was the case with our patient.

Treatment. The only definitive treatment for $\mathrm{CAB}$ is surgery. Although laparoscopic surgery has been previously demonstrated to be a possible approach in patients with small bowel obstruction, ${ }^{4,5,7-9}$ it is only safe and feasible when the bowel is not very distended and there is sufficient working space. Our patient presented with a tense abdomen, which indicated gross distension of his bowels. Therefore, laparoscopic surgery would not be practical due to limited additional space for insufflation for pneumoperitoneum. It may be attempted in patients with less marked distension, but there should be a low threshold to convert to laparotomy for the surgery to be completed swiftly and safely. 
This is a rare case of $\mathrm{CAB}$ with multiple sites of dense adhesions that resulted in the compression and entrapment of small bowel at multiple sites. $\mathrm{CAB}$ is a rare pathology especially in adults, and is difficult to diagnose as adhesion bands are radiologically occult. There must be a high index of suspicion of $\mathrm{CAB}$ in patients with virgin abdomen who present with recurrent intestinal obstruction even with unremarkable haematological and radiological findings.

\section{REFERENCES}

1. Sozen S, Emir S, Yazar FM, et al. Small bowel obstruction due to anomalous congenital peritoneal bands - case series in adults. Bratisl Lek Listy 2012;113:186-9.

2. Habib E, Elhadad A. Small bowel obstruction by a congenital band in 16 adults. Ann Chir 2003;128:94-7.

3. Akgür FM, Tanyel FC, Büyükpamukçu N, et al. Anomalous congenital bands causing intestinal obstruction in children. J Pediatr Surg 1992;27:471-3.

4. Abdelwahed Y, Saber R, Imen BI, et al. A case report of small bowel obstruction secondary to congenital peritoneal band in adult. Int J Surg Case Rep 2017;30:23-5.

5. Nicolas G, Kfoury T, Shimlati R, et al. Diagnosis and Treatment of Small Bowel Strangulation Due To Congenital Band: Three Cases of Congenital Band in Adults Lacking a History of Trauma or Surgery. Am J Case Rep 2016;17:712-9.

6. Balthazar EJ, George W. Holmes Lecture. CT of small-bowel obstruction. AJR Am J Roentgenol 1994;162:255-61.

7. Franklin ME Jr, Gonzalez JJ Jr, Miter DB, et al. Laparoscopic diagnosis and treatment of intestinal obstruction. Surg Endosc 2004; 18:26-30.

8. Al-Mulhim AA. Laparoscopic management of acute small bowel obstruction. Experience from a Saudi teaching hospital. Surg Endosc 2000;14:157-60.

9. Agresta F, Piazza A, Michelet I, et al. Small bowel obstruction. Laparoscopic approach. Surg Endosc 2000;14:154-6.

Jia Hao Law, ${ }^{1}{ }_{M B B S}$, Su Ann Lui,${ }^{1}$ MMed,

Zong Jie Koh, ${ }^{1}$ MMed (Surgery), Ning Qi Pang, ${ }^{1}$ FRCS (Edin) (Gen Surgery)

Celene Wei Qi $\underline{\mathrm{Ng}},{ }_{\text {FRCS (Edin) }}$

${ }^{1}$ Department of Surgery, National University Hospital, National University Health System, Singapore

Correspondence: A/Prof Celene Wei Qi Ng, Division of Breast Surgery, University Surgical Cluster, National University Hospital, 1E Kent Ridge Road, NUHS Tower Block Level 8, Singapore 119228.

Email: celene_wq_ng@nuhs.edu.sg 